Randomised controlled trial

\section{Elastic compression stockings fail to prevent post-thrombotic syndrome after a first deep vein thrombosis}

10.1136/eb-2014-101873

\section{Abir Kanaan}

Department of Pharmacy Practice, MCPHS University, Worcester, Massachusetts, USA

Correspondence to: Dr Abir Kanaan, Department of Pharmacy Practice, MCPHS University, 19 Foster Street, Worcester, MA 01608, USA; abir.kanaan@mcphs.edu

Commentary on: Kahn SR, Shapiro S, Wells PS, et al. Compression stockings to prevent post-thrombotic syndrome: a randomised placebo-controlled trial. Lancet 2014;383:880-8.

\section{Implications for practice and research}

- Elastic compression stockings should not be routinely used for the prevention of post-thrombotic syndrome (PTS) following a first deep vein thrombosis (DVT) episode.

- The role of elastic compression stockings in PTS prevention should be further examined.

\section{Context}

Post-thrombotic syndrome (PTS) is a chronic complication of deep venous thrombosis (DVT) characterised by clinical features ranging from minor limb swelling to leg ulceration. One in three patients with DVT will develop PTS sequelae within 5 years, with most cases reported within the first 2 years. ${ }^{1}$ Venous hypertension, resulting from valvular reflux and residual thrombus, is implicated in the pathophysiology of PTS. ${ }^{2}$ Elastic compression stockings reduce venous hypertension and have been evaluated for the prevention of PTS; however, limitations in trial design affect the generalisability of published results. ${ }^{3}$ The study by Kahn and colleagues aims to evaluate the role of compression stockings in the prevention of PTS while addressing the methodological limitations of previously published trials.

\section{Methods}

Patients with their first symptomatic proximal DVT (with or without concurrent distal DVT or pulmonary embolism) were randomised to compression stockings (30-40 mm Hg; $\mathrm{n}=410$ ) or placebo stockings ( $5 \mathrm{~mm} \mathrm{Hg}$; $\mathrm{n}=396)$. Stockings were applied within 2 weeks of DVT diagnosis and replaced every 6 months (earlier if they had torn, or if the patient's leg size had changed). Patients wore stockings for 2 years and were evaluated at baseline, 1, 6, 12, 18 and 24 months. To facilitate the diagnosis of PTS using Ginsberg's criteria, patients did not wear stockings to follow-up visits. The cumulative incidence of PTS was reported for 409 and 394 patients in the compression stockings and placebo groups, respectively, using a modified intention-to-treat Cox regression analysis, supplemented by a pre-specified per-protocol analysis of patients who reported frequent use of their allocated treatment.

\section{Findings}

Baseline characteristics between groups were similar and mean age was 55.1 years $(S D(S D)=15.5)$. Most patients received anticoagulation therapy for at least 3 months to treat their DVT. Mean time to randomisation was 4.7 days $(\mathrm{SD}=3.9)$. Groups were similar with anticoagulation and study stockings use and follow-up visits. The cumulative incidence of PTS was not statistically different between groups $(14.2 \%$ vs $12.7 \%$ for treatment and placebo respectively; $\mathrm{HR}=1.13 ; 95 \% \mathrm{CI} 0.73$ to $1.76 ; \mathrm{p}=0.58$ ). Findings suggest that compression stocking use after a first proximal DVT did not reduce the incidence of PTS, meaning routine use is not recommended.

\section{Commentary}

This study provides definitive evidence against the routine use of elastic compression stockings following a first episode of deep vein thrombosis for the prevention of post-thrombotic syndrome (PTS). These findings are not consistent with previously published trials and meta-analyses which supported the use of compression stockings in this indication. ${ }^{4-6}$ In these trials and analyses, limitations in methodology including assessment of PTS prevented generalisability of findings. However, the use of compression stockings to prevent PTS was supported by practice guidelines and continued in practice, even though their use could be challenging due to their cost and potential to cause itchiness, restriction and discomfort.

The findings by Kahn and colleagues will have an impact on current clinical practice as they are generalisable and can be extrapolated to the general population in whom the use of compression stockings would be considered. The authors employed a multicentre study design that included a large sample size, allowed a 2-year follow-up and protected against bias with randomisation and allocation concealment. Although adherence to study stockings diminished over the follow-up period, similar results between the per-protocol and intention-to-treat analyses increased confidence in the study's findings. Patients enrolled in the study also resembled previously studied populations and the compression strength used was the same as in previous trials.

While this study does not support the use of elastic compression stockings, other preventive measures can still be implemented to prevent PTS in patients diagnosed with acute DVT. These include modifying patient-specific risk factors, enhancing the time within therapeutic range and optimising duration of therapy when using anticoagulants, preventing recurrent ipsilateral DVT, and surgically intervening on venous valves. ${ }^{3}$ Although compression stockings should not be used to prevent PTS after an acute DVT, their role in treating symptoms of established PTS remains understudied and warrants further investigation.

Competing interests None.

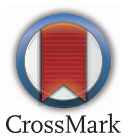

\section{References}

1. Prandoni P, Lensing AWA, Cogo A, et al. The long term clinical course of acute deep venous thrombosis. Ann Intern Med 1996;125:1-7.

2. Prandoni P, Frulla M, Sartor A, et al. Venous abnormalities and the post-thrombotic syndrome. J Thromb Haemost 2005;3:401-2.

3. Kanaan A0, Lepage JE, Djazayeri S, et al. Evaluating the role of compression stockings in preventing post-thrombotic syndrome: a review of the literature. Thrombosis 2012;2012:694851.

4. Prandoni P, Lensing AWA, Prins MH, et al. Below-knee elastic compression stockings to prevent the post-thrombotic syndrome. A randomized, controlled trial. Ann Intern Med 2004;141:249-6.

5. Aschwanden M, Jeanneret C, Koller MT, et al. Effect of prolonged treatment with compression stockings to prevent post-thrombotic syndrome: a randomized controlled trial. J Vasc Surg 2008;47:1015-21.

6. Musani MH, Matta F, Yackoub AY, et al. Venous compression for prevention of postthrombotic syndrome: a meta-analysis. Am J Med 2010;123:735-40. 